\title{
The IAA- and ABA-responsive transcription factor CgMYB58 upregulates lignin biosynthesis and triggers juice sac granulation in pummelo
}

Meiyan Shi', Xiao Liu', Haipeng Zhang ${ }^{1}$, Zhenyu He${ }^{1}$, Hongbin Yang ${ }^{1}$, Jiajing Chen', Jia Feng ${ }^{1}$, Wenhui Yang ${ }^{1}$,

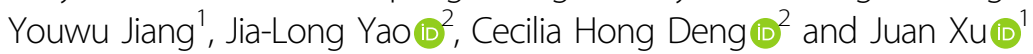

\begin{abstract}
In citrus, lignin overaccumulation in the juice sac results in granulation and an unpleasant fruit texture and taste. By integrating metabolic phenotyping and transcriptomic analyses, we found 702 differentially expressed genes (DEGs), including 24 transcription factors (TFs), to be significantly correlated with lignin content. CgMYB58 was further identified as a critical R2R3 MYB TF involved in lignin overaccumulation owing to its high transcript levels in Huanong Red-fleshed pummelo (HR, Citrus grandis) fruits. Transient expression of CgMYB58 led to an increase in the lignin content in the pummelo fruit mesocarp, whereas its stable overexpression significantly promoted lignin accumulation and upregulated 19 lignin biosynthetic genes. Among these genes, CgPAL1, CgPAL2, Cg4CL1, and CgC3H were directly modulated by CgMYB58 through interaction with their promoter regions. Moreover, we showed that juice sac granulation in pummelo fruits could be affected by indole-3-acetic acid (IAA) and abscisic acid (ABA) treatments. In HR pummelo, ABA significantly accelerated this granulation, whereas IAA effectively inhibited this process. Taken together, these results provide novel insight into the lignin accumulation mechanism in citrus fruits. We also revealed the theoretical basis via exogenous IAA application, which repressed the expression of CgMYB58 and its target genes, thus alleviating juice sac granulation in orchards.
\end{abstract}

\section{Introduction}

Lignin, which normally accumulates in secondary cell walls, has been reported to play a critical role in maintaining mechanical strength and facilitating the transport of water and nutrients in plants ${ }^{1,2}$, and is crucial for plant growth and responses to environmental stress ${ }^{3,4}$. In woody plants, a high lignin content in vascular bundles is a positive trait for woody products; however, lignin accumulation is undesirable in the fruit flesh of crop species, such as loquat (Eriobotrya japonica) ${ }^{5,6}$, pear (Pyrus bretschneideri) $^{7,8}$, and citrus ${ }^{9,10}$. Therefore,

Correspondence: Cecilia Hong Deng (cecilia.deng@plantandfood.co.nz) or Juan Xu (xujuan@mail.hzau.edu.cn)

${ }^{1}$ Key Laboratory of Horticultural Plant Biology, Ministry of Education, Wuhan, Hubei 430070, China

${ }^{2}$ The New Zealand Institute for Plant and Food Research Limited, Private Bag

92169, Auckland 1142, New Zealand understanding the regulatory mechanism underlying lignin biosynthesis and lignin accumulation is of great importance, and such knowledge could potentially be applied to assist fruit breeding programs.

In plants, the lignin biosynthetic pathway involves a series of enzymes, including phenylalanine ammonia lyase (PAL), cinnamate 4-hydroxylase $(\mathrm{C} 4 \mathrm{H})$, 4coumarate CoA ligase (4CL), hydroxy cinnamoyl CoA, shikimate/quinate hydroxy cinnamoyl transferase (HCT), p-coumarate 3-hydroxylase $(\mathrm{C} 3 \mathrm{H})$, caffeoyl $\mathrm{CoA}$ 3-Omethyltransferase (CCoAOMT), cinnamoyl CoA reductase (CCR), caffeic acid $O$-methyltransferase, cinnamyl alcohol dehydrogenase, and ferulate 5-hydroxylase. Through reactions catalyzed by these enzymes, plants produce three types of lignin monomers, $\mathrm{p}$ hydroxyphenyl $(\mathrm{H})$ monomers, guaiacyl $(\mathrm{G})$ monomers, 
and syringyl (S) monomers, which are then polymerized by laccases (LACs) or peroxidase $e^{2,3,11}$.

Transcription factors (TFs) regulating lignin biosynthetic genes have been well studied in various plant species, such as Arabidopsis thaliana ${ }^{12}$, Populus trichocarpa ${ }^{13}$, and switchgrass (Panicum virgatum) ${ }^{14}$. In Arabidopsis, AC elements bound specifically by MYBs are widely distributed in the promoters of PAL1, PAL2, 4CL1, 4CL2, HCT, C3H1, CCOAOMT1, CCR1, and CAD5 ${ }^{2}$. AtMYB58 and AtMYB85 selectively bind AC elements in the promoter of $4 C L 1$, thereby regulating developmental lignification in vascular tissues ${ }^{15,16}$. Evidence has confirmed that MYBs can regulate lignin biosynthetic genes involved in secondary cell wall formation, including PvMYB58/63, PvMYB42/85, and PvMYB4, in switchgrass $^{14}$. EjMYB1, a homolog of AtMYB58 in loquat, has been reported to modulate lignin biosynthesis during fruit storage $^{5}$. In the stone cells of pear fruits, PbrMYB169 was found to upregulate lignification by activating the promoters of lignin biosynthetic genes ${ }^{7}$. In addition to MYBs, other TFs have also been revealed to play important roles in fruit lignin biosynthesis; e.g., EjNAC1, EjAP2-1, and EjHSF3 are involved in lignin formation in loquat ${ }^{6,17,18}$.

Citrus fruits, which are the most popular fruits worldwide, provide an assortment of functional components that are beneficial to human health, including flavonoids ${ }^{19,20}$, volatile compounds ${ }^{21}$, alkaloids ${ }^{22}$, and carotenoids $^{23-26}$. However, in citrus fruit juice sacs, overaccumulation of lignin (a physiological disorder known as granulation) often occurs ${ }^{9,10,27,28}$. This granulation causes a "gritty" texture and dryness of the juice sacs, reduces consumer acceptance of the fruits, and is a severe problem in sweet orange (Citrus sinensis) ${ }^{10}$, Ponkan mandarin $(C \text {. reticulata })^{28}$ and Guanximiyou pummelo (C. grandis) ${ }^{9}$. Granulation is affected by disorders involving mineral nutrition, phytohormones, growth temperature, and genetic factors ${ }^{29}$. Previous findings have revealed that TFs play a crucial role in sweet orange granulation. For example, CsMYB330 and CsMYB85, which are homologs of AtMYB58/AtMYB63 and AtMYB85, respectively, have been reported to interact with CsMYB308 through the binding of the Cs4CL1 promoter $^{10,30}$ to regulate juice sac granulation.

Among citrus fruits, pummelo fruits are especially prone to granulation during ripening and storage? However, our knowledge of the regulation of lignin accumulation and thus granulation is limited in pummelo fruits. In the present study, we investigated juice sac granulation and evaluated lignin accumulation in four pummelo cultivars, Huanong Red-fleshed (HR), Hirado Buntan (HB), Fenghuang (FH), and Kao Pan (KP), throughout different fruit developmental stages. We found that indole-3-acetic acid (IAA)- and abscisic acid (ABA)-responsive CgMYB58 played a critical role in lignin biosynthesis. This TF can interact with the promoter regions of CgPAL1, CgPAL2, Cg4CL1, and CgC3H. These results contribute to our understanding of the genetic mechanism controlling juice sac granulation and provide a potential practical approach to inhibiting this phenomenon in citrus orchards.

\section{Results \\ Lignin excessively overaccumulated during juice sac granulation in pummelo}

We investigated juice sac granulation in four pummelo cultivars, $\mathrm{HR}, \mathrm{HB}, \mathrm{FH}$, and $\mathrm{KP}$, at 205 days post anthesis (DPA) and found that HR exhibited the most severe granulation (Fig. 1a). Moreover, the granulated juice sacs of HR were stained deep red with the lignin-specific stain phloroglucinol- $\mathrm{HCl}$, whereas the other three pummelo cultivar fruits showed only very small red spots or were colorless after staining (Fig. 1b, c). The subsequent examination of paraffin-embedded sections showed that the cell walls of the HR juice sacs were stained red and purple by phloroglucinol- $\mathrm{HCl}$ and toluidine blue, respectively, and were thickened, owing to the excessive accumulation of lignin (Fig. 1d, e).

Next, we measured the lignin contents of the juice sacs of these four cultivars at 55,85, 115, 145, 175, and 205 DPA (collected in 2016). The lignin content of the juice sacs gradually increased in HR during all developmental stages and the lignin content in HR was much higher than that in $\mathrm{KP}, \mathrm{FH}$, and $\mathrm{HB}$ at 175 and $205 \mathrm{DPA}$ (Fig. 1f). The lignin accumulation in the juice sacs of HR sharply increased from 145 to $205 \mathrm{DPA}$ and reached $5.69 \times 10^{3}$ A280 $\mathrm{kg}^{-1}$ at 205 DPA (Fig. 1f). Moreover, we assessed the lignin contents of nine pummelo cultivars at $\sim 200$ DPA (collected in 2014). The data showed that HR contained the highest lignin content among all tested cultivars, whereas the others maintained lower levels of lignin, with an average of $0.6 \times 10^{3} \mathrm{~A} 280 \mathrm{~kg}^{-1}$ (Supplementary Fig. S1). Highly significant lignin accumulation was also observed in San Hong (a bud mutant of Guanximiyou) (Supplementary Fig. S2a, b), which has been reported to readily undergo juice sac granulation ${ }^{9}$. Taken together, these results indicated that the juice sac granulation of pummelo resulted from the overaccumulation of lignin.

\section{Transcriptomic and qRT-PCR analyses revealed that CgMYB58 modulates lignin biosynthetic genes}

To explore the mechanism of granulation in $\mathrm{HR}$, we performed transcriptomic analyses using the juice sacs of HR and two controls (HB and KP) at 55, 85, 115, 145, and 205 DPA. The RNA sequencing (RNA-seq) approach produced $\sim 6 \mathrm{~Gb}$ of data for each sample and $\sim 92 \%$ of the reads were mapped to the pummelo genome ${ }^{31}$ (Supplementary Table S1). Principal component analysis of fragments per kilobase of transcript per million mapped 


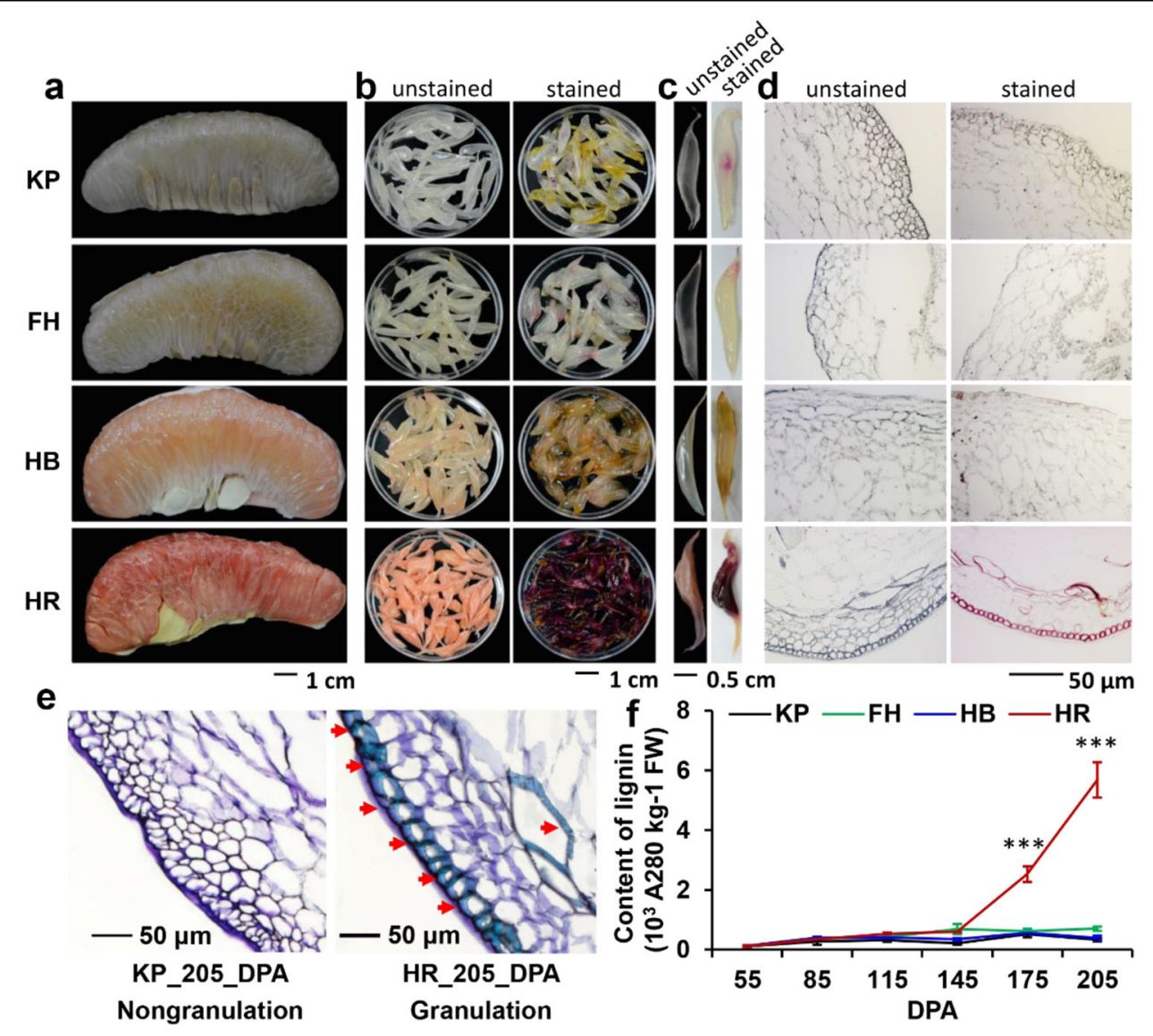

Fig. 1 HR fruits accumulated high lignin content in their juice sacs at late developmental stages. a The juice sacs of four pummelo cultivars, Kao Pan (KP), Fenghuang (FH), Hirado Buntan (HB), and Huanong Red-fleshed (HR), were collected from mature fruits at 205 DPA. b The juice sacs were separated (left) and stained with phloroglucinol hydrochloride dye (right). The high lignin contents in the HR juice sacs resulted in a dark red color $(\mathbf{b}, \mathbf{c})$. $\mathbf{d}$ The HR juice sacs were stained dark red and their sections were stained red, indicating a relatively high level of lignin accumulation. The bar applies to all the images in the same panel. e Paraffin sections of KP and HR juice sacs were stained with toluidine blue. The arrowheads point to blue-stained thickened cell walls with a high lignin content. $\mathbf{f}$ The lignin content in the juice sacs of four pummelo cultivars was measured at six developmental stages. In HR, the lignin content increased significantly and accumulated much more than that in the other cultivars after 145 DPA. The asterisks indicate significant differences in HR compared with KP, FH, and HB at 175 and 205 DPA, and were analyzed by Duncan's multiple comparison test $\left.{ }^{* * *} P<0.05\right)$

reads (FPKMs) and differentially expressed gene (DEG) analyses were performed (Supplementary Fig. S3), allowing us to further analyze DEGs enriched in the biosynthesis of secondary metabolites (Supplementary Fig. S4a) and phenylpropanoid metabolites (Supplementary Fig. S4b) by KEGG (Kyoto Encyclopedia of Genes and Genomes) analysis.

Owing to the lignin content being greatly different between HR and the other pummelo cultivars, the FPKMs of lignin biosynthetic genes were analyzed (Fig. 2 and Supplementary Table S2). The transcript levels of the following genes sharply increased from 145 DPA to 205 DPA in HR: CgPAL1, $-2,-3,-4$, and -5 ; $C g C 4 H$; Cg4CL1; $\mathrm{CgC3H}$; $\mathrm{CgCCoAOMT3}$ and -4 ; and CgLAC4, $-5,-7,-11,-13,-15$, and -22 (Fig. 2). The real-time quantitative reverse-transcription PCR (qRT-PCR) results for CgPAL1, CgPAL2, Cg4CL1, and $\mathrm{CgC3H}$ were consistent with the FPKM results (Supplementary Fig. S5 and Supplementary Table S2).

According to the juice sac granulation phenotype and lignin accumulation pattern (Fig. 1), we then selected the total lignin content as a bait for assessing the correlation with genes from the transcriptome. The genes correlated with the total lignin content with an absolute coefficient $\geq$ 0.75 were chosen as candidates. A total of 702 genes were found to be related to the total lignin content (Supplementary Table S3). According to GO enrichment analysis, the genes corresponding to lignin content were significantly enriched in the phenylpropanoid pathway and the lignin catabolic/metabolic pathway (Supplementary Fig. S6). Among these genes, 24 TFs were identified, which were abundant in transcripts in HR at 145 DPA and 


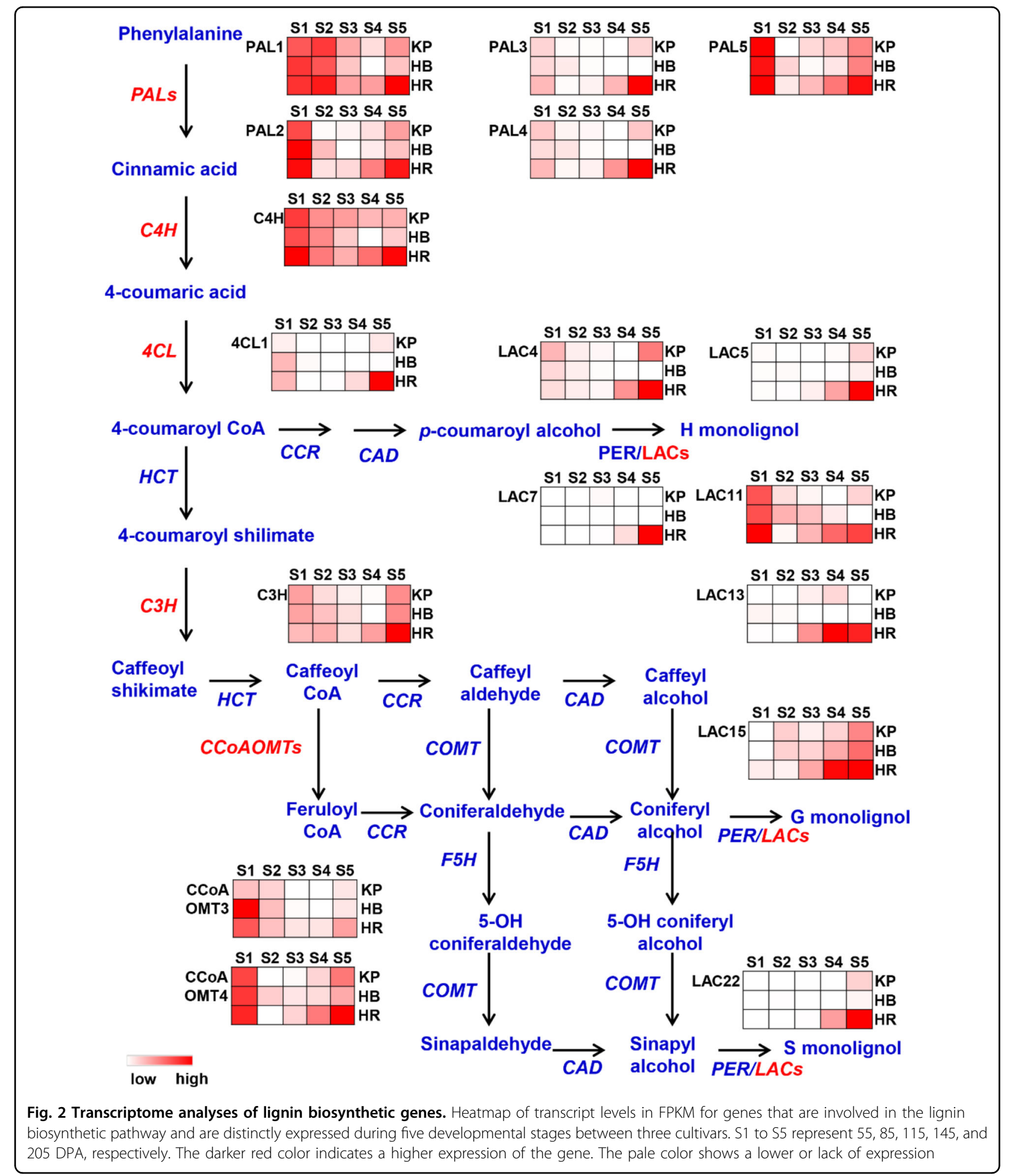

205 DPA (Fig. 3a), and exhibited a relatively strong correlation (coefficient $\geq 0.8$ ) with 12 lignin biosynthetic genes (Fig. 3b). The qRT-PCR results for CgMYB46, CgMYB44, CgMYB112, and CgbHLH95 were also consistent with the FPKM results (Supplementary Fig. S5 and
Supplementary Table S2), and the expression pattern of these TFs that were highly expressed in HR at the examined stages was in agreement with the high lignin content. Remarkably, homologous genes of CgMYB58, $C g M Y B 46$, and $C g S N D 1$ have been previously reported to 

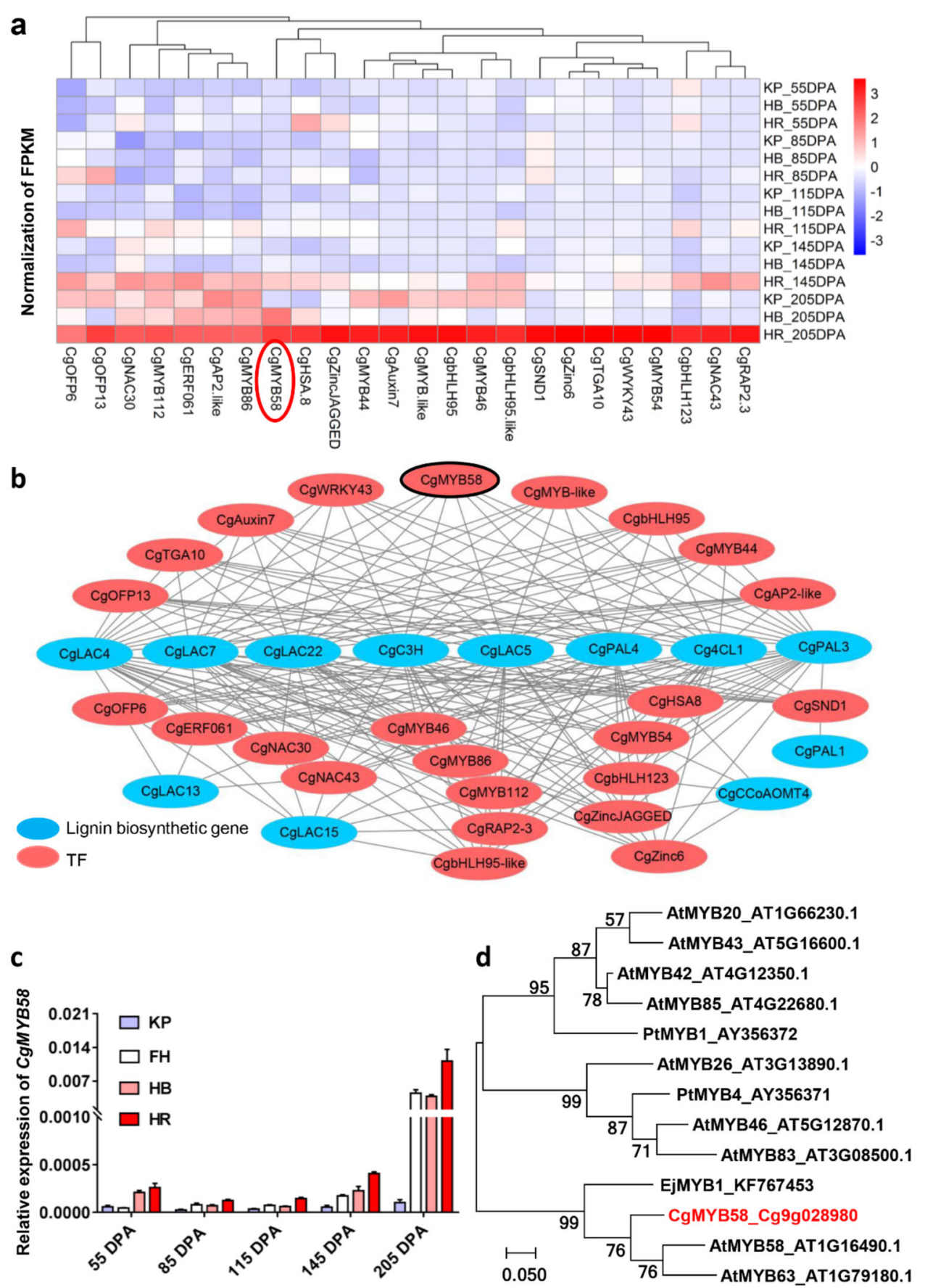

Fig. 3 Transcriptome analyses of associated transcription factors. a Heatmap of transcription factors (TFs) involved in lignin biosynthesis. $\mathbf{b}$ Gene expression networks showing TFs (potential regulators) and genes encoding lignin biosynthetic enzymes. The black lines indicate that the coefficient value between the node genes is higher than 0.8. c Expression level of CgMYB58 in KP, FH, HB, and HR at 55, 85, 115, 145, and 205 DPA. DPA: Days post anthesis. The housekeeping gene used was described in a previous report ${ }^{26}$. $\mathbf{d}$ Phylogenetic analysis of CgMYB58 and related proteins from other plant species. The scale bar represents 0.05 substitutions per site. The red color indicates CgMYB58

modulate secondary cell wall synthesis in Arabidopsis $^{15,16,32}$. We showed that CgMYB58 exhibited a higher transcript level than did CgMYB46 and CgSND1 in HR at 205 DPA (Supplementary Table S2). Our qRT-PCR assay revealed that $C g M Y B 58$ was significantly highly expressed in $\mathrm{HR}$ compared with $\mathrm{KP}, \mathrm{FH}$, and $\mathrm{HB}$ throughout all developmental stages (Fig. 3c). In addition, CgMYB58 was also highly expressed in San Hong 
granulated juice sacs and Guanxi red-fleshed pummelo in association with granulation and high Cg4CL1 expression (Supplementary Fig. S2b-e). CgMYB58 was closely related to AtMYB58 and EjMYB1, as confirmed by phylogenetic analysis (Fig. 3d) and amino acid sequence alignment (Supplementary Fig. S7a); these proteins have been previously shown to regulate lignin biosynthesis in Arabidopsis $^{16}$ and loquat ${ }^{5}$. Subcellular localization analysis revealed that CgMYB58 localized to the nucleus of citrus leaf protoplast cells (Supplementary Fig. S7b). Overall, we found that 702 DEGs were correlated with lignin accumulation, and that CgMYB58 was probably the key TF involved in lignin biosynthesis in pummelo fruits.

\section{Overexpression of CgMYB58 promoted the accumulation of lignin in citrus tissues}

The coding sequence of CgMYB58 was identical among $\mathrm{HR}$ and other low-lignin-accumulating pummelo cultivars and thus was named CgMYB58-1 (Supplementary Table S4). Therefore, in the following functional verification experiments, CgMYB58-1 was used, as it is the dominant transcript expressed in HR fruit. To simplify, we replaced CgMYB58-1 with $C g M Y B 58$. In addition, the promoter sequences of $C g M Y B 58$ were mainly conserved among the pummelo cultivars but differed from those of sweet orange, lime (C. aurantifolia), and mandarin by two indels (Supplementary Table S4).

To verify the biological function of CgMYB58 in lignin accumulation, we first transiently expressed $C g M Y B 58$ in the mesocarp of KP and HR fruits. The results emphasized that $C g M Y B 58$ promoted lignin accumulation in the mesocarp of both pummelo cultivars (Fig. 4a, b). Wildtype (WT) Red Marsh (RM) calli were stably transformed with a CgMYB58 overexpression (CgMYB58-OE) construct. The combined analyses of the levels of $C g M Y B 58$ expression and lignin contents in eight CgMYB58-OE lines (Fig. 4c) indicated that, compared with the WT, seven of these lines exhibited a significantly higher lignin content (Fig. 4d). Furthermore, they were stained with phloroglucinol-HCl; the selected CgMYB58-OE calli lines displayed a deep red color, whereas the WT calli remained pale yellow (Fig. 4e). RNA-seq analysis was also carried out using two stable CgMYB58-OE lines and WT-RM calli, with three replicates analyzed for each sample. A total of 397 genes were found to be upregulated in CgMYB58-OE4 and CgMYB58-OE11 compared to the WT, whereas 372 were downregulated (Supplementary Fig. S8). The transcript levels of most genes involved in the lignin biosynthetic pathway were greatly altered (Fig. 4f), with 19 significantly increased in the CgMYB58-OE lines (Fig. 4f and Supplementary Table S5). Collectively, these data showed that overexpression of CgMYB58 was responsible for lignin accumulation in citrus.

\section{CgMYB58 modulated lignin accumulation through interactions with the promoters of four lignin biosynthetic genes}

To verify whether CgMYB58 could regulate lignin biosynthetic genes in citrus, the promoters of CgPAL1, CgPAL2, Cg4CL1, and $C g C 3 H$ were cloned and sequenced. AC elements (5'-ACCAA(T)CC-3' or $5^{\prime}$ ACCTAAC- $3^{\prime}$ ) were identified in the promoter sequences at $-383,-248$, and $-210 \mathrm{bp}$ from the translational start codon for CgPAL1; at -500 and -295 bp for CgPAL2; at -224 bp for Cg4CL1; and at $-1966,-1954,-1415,-457$, -405 , and -15 bp for $\mathrm{CgC3H}$ (Supplementary Table S6). To determine whether CgMYB58 could bind to these promoters, a dual luciferase (LUC) transcriptional activity assay was performed in tobacco leaves. A 35S:CgMYB58 construct and an empty vector were used as effectors, whereas the promoters of CgPAL1, CgPAL2, Cg4CL1, and $\mathrm{CgC} 3 \mathrm{H}$ were fused as reporters. The relative LUC activity derived from these promoters was strongly stimulated by CgMYB58 (Fig. 5a). Yeast one-hybrid assays (Y1Hs) then revealed that $\mathrm{CgMYB} 58$ could interact with the promoters of $C g P A L 2$ and $C g C 3 H$ (Fig. 5b). Notably, the promoter of Cs4CL1 of sweet orange was also bound by CsMYB330, a homolog of CgMYB58, as shown in a previous $\mathrm{Y} 1 \mathrm{H}$ assay $^{10}$. As the promoter sequences of $4 C L 1$ and the coding sequence of MYB58 were conserved between sweet orange and pummelo, the results were identical when using gene sequences from pummelo and thus were not presented here. Finally, using a pMAL-c5x-CgMYB58 fusion protein, we performed an electrophoretic mobility shift assay (EMSA) to verify these interactions. The purified pMAL-c5x-CgMYB58 protein could directly bind to the 25-27 bp probes containing the $\mathrm{AC}$ elements in the promoters of $C g P A L 1, C g P A L 2, C g 4 C L 1$, and $C g C 3 H$ (Fig. 5c). These results demonstrated that CgMYB58 promoted the lignification process by directly upregulating the expression of four monolignol biosynthetic genes: CgPAL1, CgPAL2, Cg4CL1, and $\mathrm{CgC} 3 \mathrm{H}$.

\section{Lignin accumulation in response to IAA and $A B A$ treatments}

The involvement of IAA and ABA in the lignification process has been investigated previously in horticultural plants $^{33,34}$. In addition, we identified ABA-responsive elements in the promoters of CgMYB58, CgPAL1, $C g P A L 2$, and $C g 4 C L 1$, whereas an auxin-responsive element was identified in the promoter of $\mathrm{CgC} 3 \mathrm{H}$ (Supplementary Fig. S9). This raised the question of whether IAA and $A B A$ could affect lignin accumulation in pummelo. Hence, we applied IAA and ABA treatments to KP, HB, and $H R$ juice sacs during 50 days of continuous culture (Fig. 6a, b). Under IAA treatment, we measured a decrease in the lignin content in the HR juice sacs, whereas under 
a
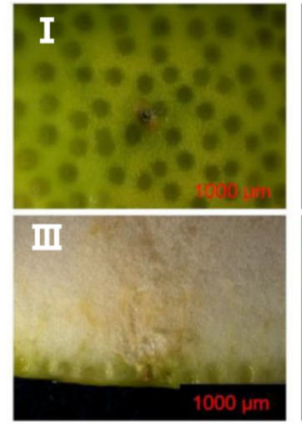

C

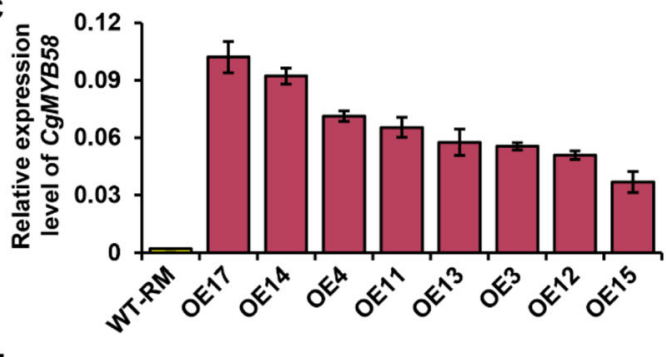

d

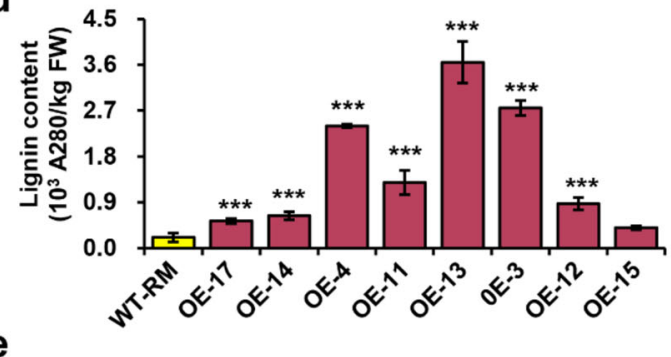

e

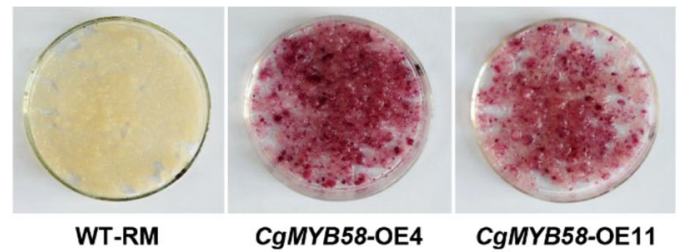

b

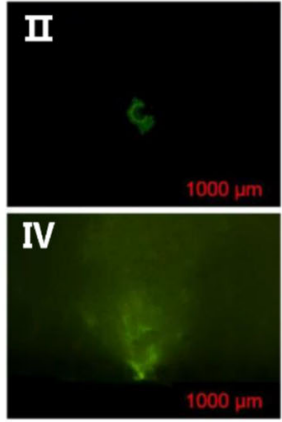

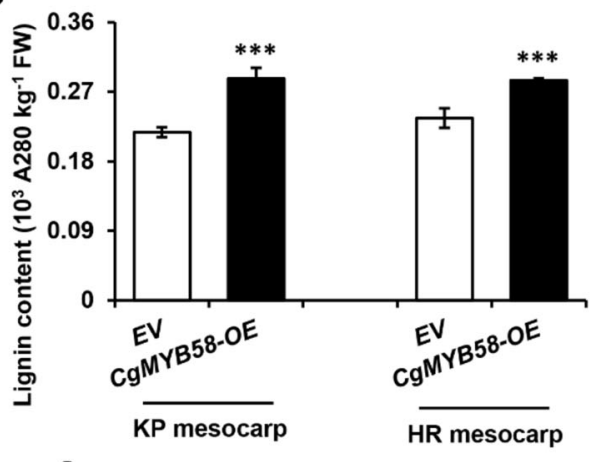

f

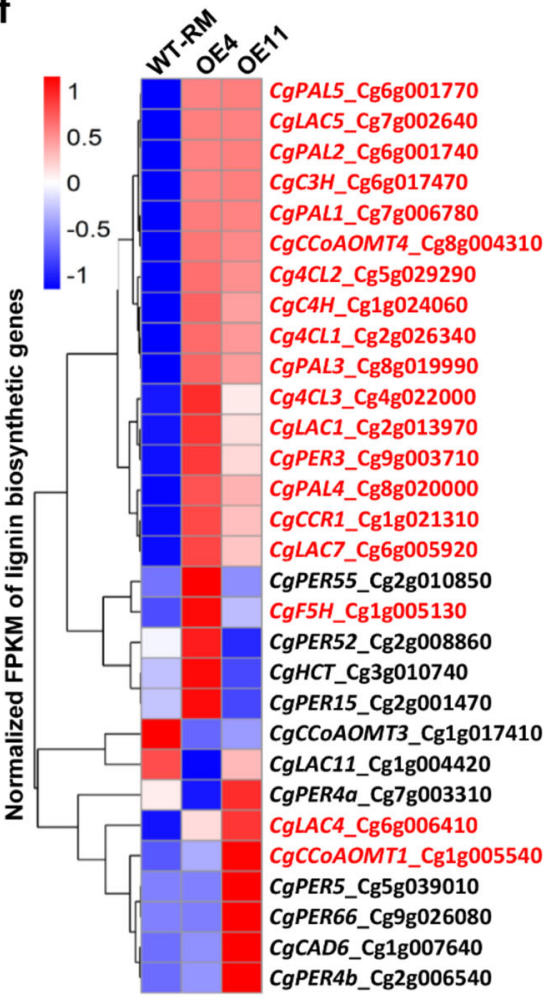

Fig. 4 Overexpression of CgMYB58 increased lignin accumulation and promoted the expression of lignin biosynthetic genes in citrus calli. a CGMYB58 transiently expressed in pummelo mesocarp. HR fruit mesocarp were injected with Agrobacteria containing an empty vector (I, II) or a CgMYB58 overexpression vector (III, IV) and imaged under bright-field (I, III) and GFP fluorescence conditions (II, IV). GFP fluorescence indicated that the vectors were successfully transferred into the pummelo fruit mesocarp. $\mathbf{b}$ Lignin contents were measured in pummelo fruit mesocarp tissue after transient transformation with an empty vector (EV) or a CgMYB58 overexpression vector. c Relative expression levels of CgMYB58 in WT-RM (Red Marsh) and eight transgenic RM calli lines overexpressing CgMYB58. d Lignin content in the calli of WT and eight transgenic lines. The asterisks indicate significant differences relative to WT-RM according to a t-test ( ${ }^{* *} P<0.05$ ). e Phenotypes WT-RM calli and the two transgenic lines stained with phloroglucinol-HCl. The WT-RM calli did not show any staining, whereas the calli of the two transgenic RM lines (CgMYB58-OE04, -OE11) were stained red. $\mathbf{f}$ Cluster heatmap based on the transcript level of lignin biosynthetic genes. Among the 30 examined lignin biosynthetic genes, 19 were upregulated in the CgMYB58-OE lines, which are highlighted in red

ABA treatment, the lignin content increased and visible granulation was observed in the HR juice sacs (Fig. 6a-c). The expression levels of CgMYB58 and its downstream genes were subsequently assessed using qRT-PCR (Fig. 6d, e). Taken together, the results showed that IAA treatment of juice sacs repressed the expression of CgMYB58 and its target genes related to lignin biosynthesis, but ABA treatment promoted their expression, suggesting that $C g M Y B 58$ responds to IAA and ABA in the regulation of the lignification process in citrus.

\section{Discussion}

Previous evidence from the commercial pummelo cultivar Guanximiyou has shown that granulation due to 


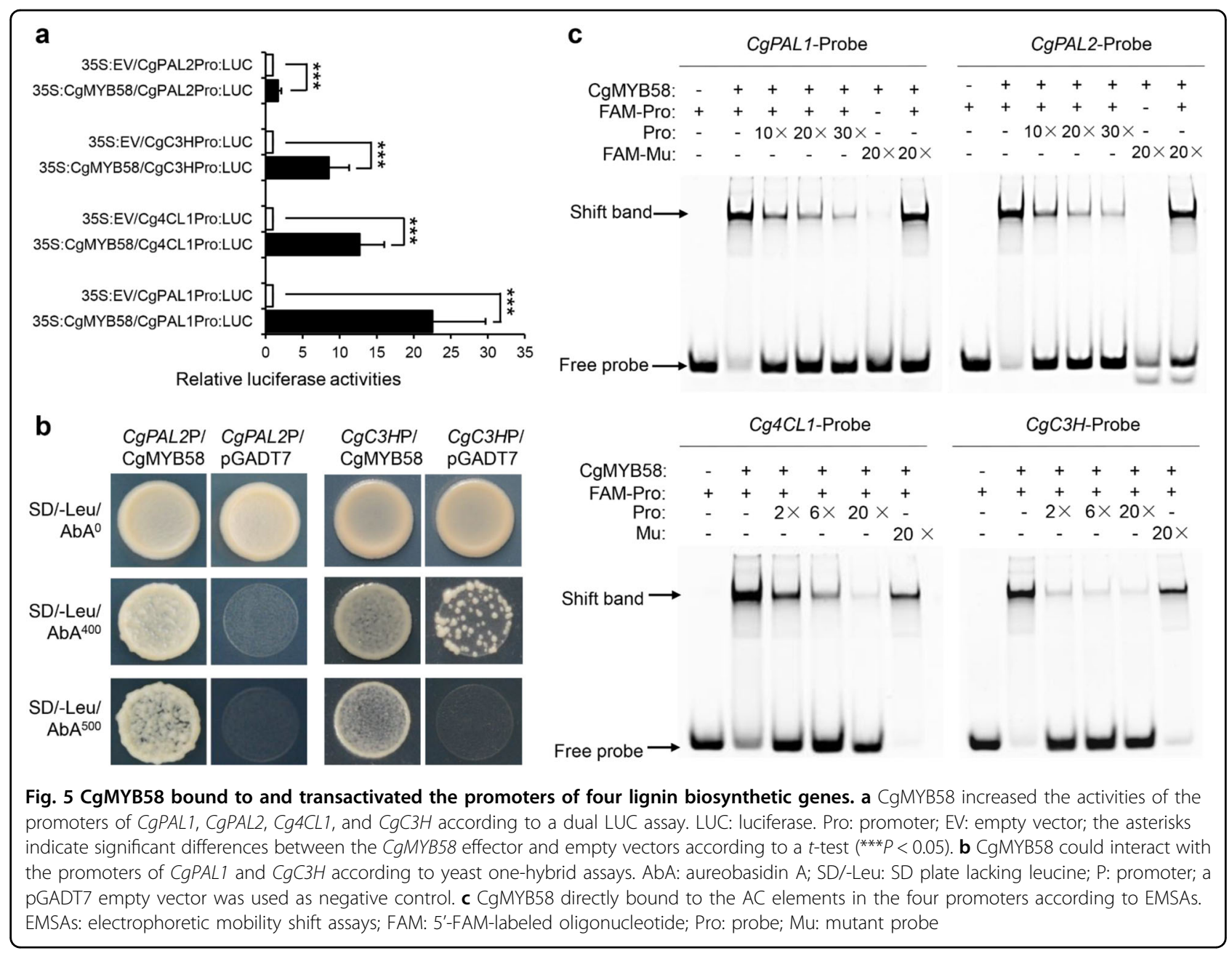

excessive lignin accumulation can greatly damage fruit sensory quality during the post-harvest process ${ }^{9}$. We therefore investigated the correlation between juice sac granulation and lignin overaccumulation in pummelo. We found that severe granulation occurred in HR juice sacs, which contained higher levels of lignin in comparison with those of other pummelo cultivars. It is likely to be that red- and non-red-fleshed citrus fruit undergo lignification during long-term storage ${ }^{9,10}$ or in response to stress related to light, water, and temperature. However, under normal physiological conditions, our study showed that a high expression level of CgMYB58 in HR was involved in lignin accumulation leading to granulation.

Lignin accumulation is crucial for plant developmental cues and responses to environmental stress in loquat fruit $^{6}$, Populus ${ }^{35}$, and cotton (Gossypium spp.) ${ }^{36}$. Several types of TFs, such as MYBs, NACs, and ERFs, have been reported to regulate lignin biosynthesis genes in plants ${ }^{6,12,13}$. In Arabidopsis, AtMYB58 and AtMYB85 selectively bind to AC elements to regulate lignification in vascular tissues ${ }^{15,16}$. In this study, by integrating transcriptome and metabolite analyses, we found that CgMYB58 promoted the expression of four upstream core monolignol biosynthetic genes by directly binding to $\mathrm{AC}$ elements in the promoters of these genes. A homolog of CgMYB58 in sweet orange, CsMYB330, was also identified as being abundant in granulated flesh, together with its target gene $C s 4 C L^{10}$. Our LUC assays and EMSAs showed that CgMYB58 could regulate not only $C g 4 C L 1$ but also three other genes (CgPAL1, $C g P A L 2$, and $C g C 3 H$ ) involved in lignin biosynthesis (Fig. 4). Notably, the PAL1, PAL2, and $C 3 H$ genes have never been reported to be directly regulated by MYB58 in horticultural species. Moreover, CgMYB58 also upregulated downstream genes, such as $L A C 1,-4,-5$, and -7 (Fig. 4 and Supplementary Table S5), suggesting that its function is broader than that of lignin biosynthetic genes than was previously thought ${ }^{10}$. Interestingly, CgMYB58 was also highly expressed in granulated San Hong juice sacs and in Guanxi red-fleshed pummelo fruits (Supplementary Fig. S2), which is consistent with the expression level of $C g 4 C L 1$. Therefore, our research provides a better 


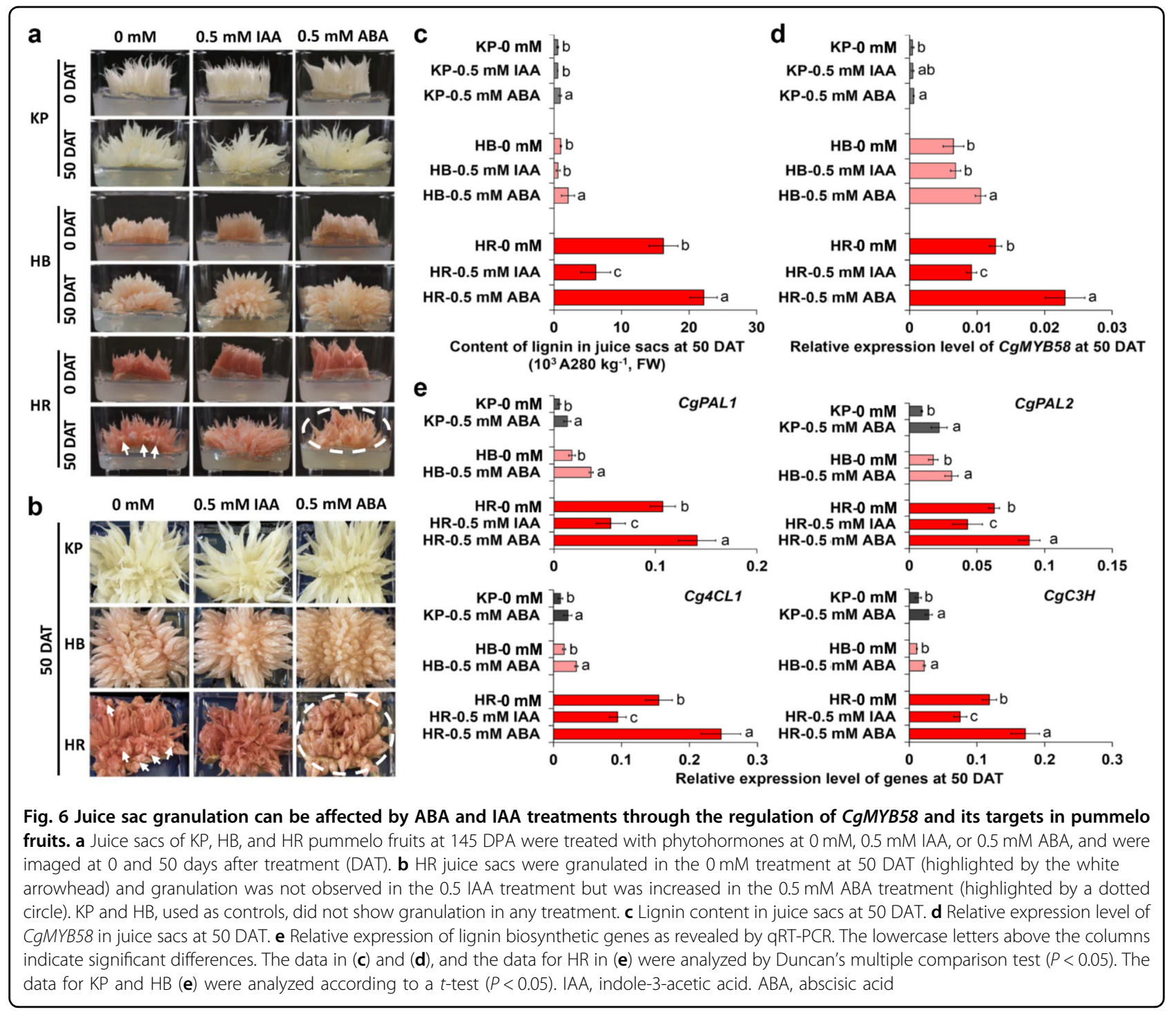

understanding of the regulation of multiple lignin biosynthetic genes by MYB58, suggesting that MYB58 is probably a TF marker responsible for granulation in citrus.

In addition to the high transcript level of CgMYB58 in HR juice sacs, the other 23 TFs were significantly correlated with lignin content (Supplementary Table S2). Among these TFs, MYB46 and SND1 have been reported to regulate secondary cell wall-related processes ${ }^{15,32}$. In Arabidopsis, AtMYB46 has been reported to directly regulate AtMYB58 in lignin biosynthesis and AtSND1 is the upstream gene of AtMYB46 ${ }^{16,32}$. Compared with CgMYB46 and CgSND1, CgMYB58 showed a higher expression level and a distinct expression pattern among HR and the other three pummelo cultivars at all developmental stages (Fig. 3c and Supplementary Table S2). Considering the RNA-seq results and previous research,
CgMYB58 is thus the critical TF regulating lignin biosynthesis in pummelo. In addition, MYB58 and three other TFs, MYB4, MYB42, and WRKY12, were reported to affect secondary cell walls through the regulation of lignin in switchgrass ${ }^{14}$. Overall, to construct a regulatory network in the future, CgMYB58 can essentially be used as a bait to retrieve interacting regulators to help construct the hierarchical regulatory network and therefore comprehensively analyze the lignin accumulation leading to citrus granulation.

In addition, previous reports have revealed that some granulated citrus cultivars ${ }^{9,10,28}$ and Luoyangqing loquat ${ }^{5,37}$ accumulate high levels of both lignin and carotenoids. In our study, lignin showed high accumulation in granulated HR with the reddest flesh (Fig. 1), which also contained high levels of total and red carotenoids. This raised the question of whether specific carotenoids 


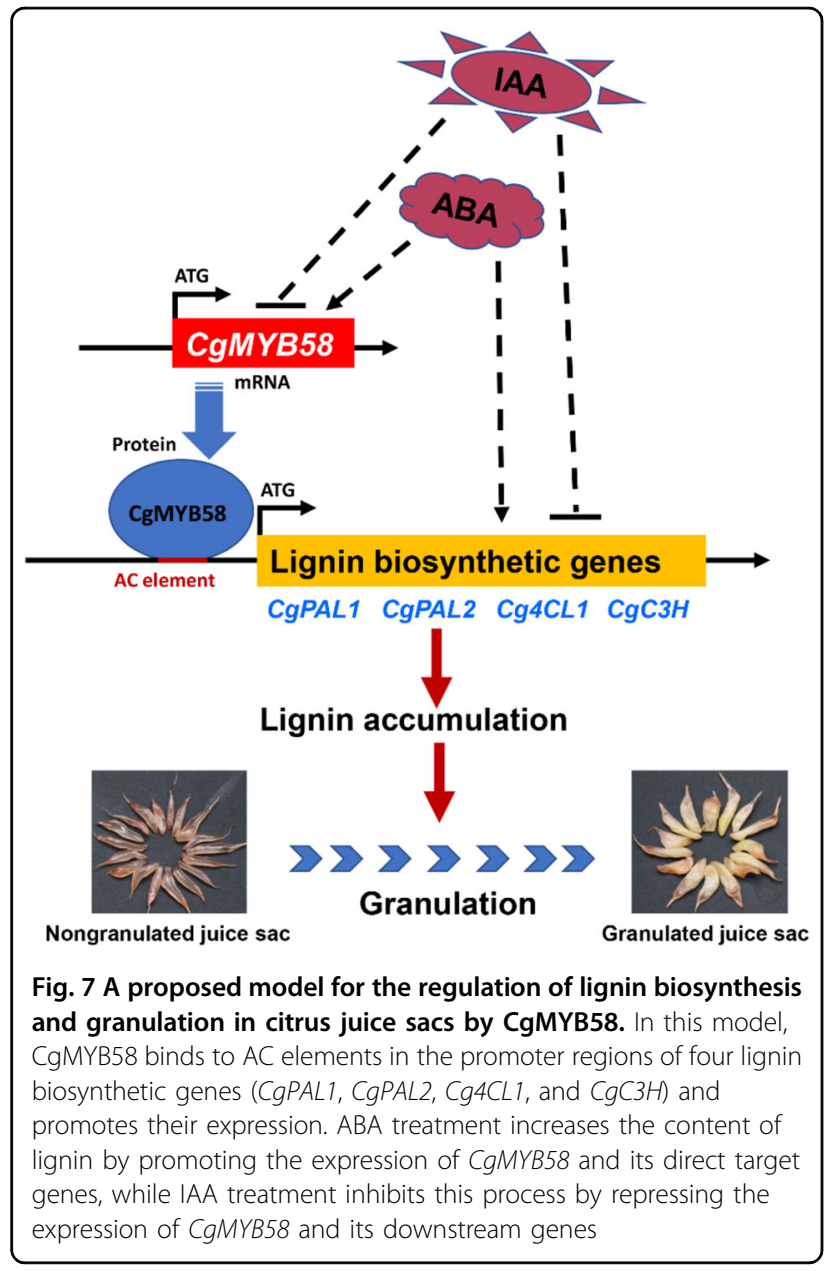

or their downstream metabolites are involved in the lignification process in HR. ABA is one of the downstream metabolites of carotenoids. Our previous reports showed that the ABA concentration was higher in pale-fleshed Feicui pummelo fruits than in red-fleshed Chuhong pummelo fruits at $150 \mathrm{DPA}^{25}$. In addition, $\mathrm{ABA}$ has been reported to be involved in the regulation of lignin biosynthetic genes and TF regulators that respond to the lignin accumulation process in plants ${ }^{34}$. Accordingly, we found that juice sacs exhibited increases in lignin content and increases in the expression levels of CgMYB58 and its target genes after being treated with ABA (Fig. 6b-d). Thus, ABA induced lignin biosynthesis by promoting the expression of CgMYB58 and its target genes in HR, HB, and KP juice sacs (Fig. 7).

IAA has been shown to influence anthocyanin biosynthesis in apple ${ }^{38}$ and to disrupt the lignification process in the roots of Asparagus officinalis ${ }^{33}$. In this study, we first determined that IAA could significantly repress lignin biosynthesis in pummelo juice sacs, further inhibiting juice sac granulation (Fig. 7). Therefore, to avoid an unpleasant sensory texture, the application of exogenous
IAA might downregulate the expression of $C g M Y B 58$ and thus relieve granulation in the juice sacs, which provides a potential approach for regulating the granulation process by applying auxin-like plant growth regulators in the field.

\section{Materials and methods \\ Plant materials}

Fruits of HR pummelo, HB pummelo, FH pummelo, and KP pummelo were collected at 55, 85, 115, 145, 175, and 205 DPA from the National Citrus Breeding Center at Huazhong Agricultural University (Wuhan, China). In addition, fruits of nine pummelo cultivars at the commercially mature stage ( 200 DPA) were also harvested (Supplementary Table S7). For each cultivar, 15-18 fruits were picked from at least three different healthy trees and divided randomly into three replicates. The juice sacs from each sample were immediately separated, frozen in liquid nitrogen, and then stored at $-80^{\circ} \mathrm{C}$.

Paraffin section observations of cell walls of the juice sacs

The reagents used for paraffin sectioning were analytically pure and purchased from Dingguo, Co. (China). The method was performed according to the methods of $\mathrm{Li}$ et $\mathrm{al}^{39}$. The sections were stained with $1 \%$ phloroglucinolhydrochloric acid or $0.1 \%$ toluidine blue solution for 3 min and then washed with $95 \%$ ethanol or deionized water for $10 \mathrm{~min}$. The sections were subsequently examined and imaged using a Zeiss Axioscope A1 microscope (Swift Microscope World, California, USA) with a $\times 0.5$ optical adapter. The images were captured and exported using ZEN 2.3 software (Zeiss).

\section{Lignin extraction and evaluation}

According to the methods of $\mathrm{Xu}$ et $\mathrm{al}^{5}{ }^{5}$ (with minor modifications), the juice sacs (or calli) were ground into powder and $8 \mathrm{~g}$ (or $10 \mathrm{~g}$ for calli) of each sample was homogenized in $15 \mathrm{ml}$ of washing buffer $(100 \mathrm{mM}$ $\mathrm{K}_{2} \mathrm{HPO}_{4} / \mathrm{KH}_{2} \mathrm{PO}_{4}, 0.5 \%$ Triton X-100, 0.5\% PVP-K30; $\mathrm{pH}$ 7.8). The mixture was washed on a shaker (GS-20, MiuLab, Hangzhou, China) at room temperature at 250 r.p. $\mathrm{m} . / \mathrm{min}$ for $30 \mathrm{~min}$ and then centrifuged $\left(5000 \times g, 25^{\circ} \mathrm{C}\right)$ for 10 min (Avanti J-26 XP, Beckman Coulter, California, USA). Finally, the supernatant was discarded. Washing buffer was added three times, as described above. Next, the precipitates were washed with $20 \mathrm{ml}$ of $100 \%$ methanol four times. The precipitates were subsequently dried at $60^{\circ} \mathrm{C}$ in a vacuum rotary evaporator (5305FG924683, Eppendorf, Germany) overnight. Fifty milligrams (mg) of the lyophilized powder of the juice sac (for calli, $100 \mathrm{mg}$ ) was dissolved in $5.0 \mathrm{ml}$ of $2.0 \mathrm{M} \mathrm{HCl}$ and $0.5 \mathrm{ml}$ of thioglycolic acid. The mixture was then boiled within a water bath at $100^{\circ} \mathrm{C}$ (LTT-600, Longyue, Shanghai, China) for $8 \mathrm{~h}$, cooled on ice for $5 \mathrm{~min}$, and centrifuged at $8000 \times g$ for $20 \mathrm{~min}$ at $4{ }^{\circ} \mathrm{C}$ (H1850R, Hunan, China). The 
precipitates were washed with distilled water three times and dried thereafter at $60^{\circ} \mathrm{C}$ in a vacuum rotary evaporator overnight. The sample was resuspended in $1.5 \mathrm{ml}$ of $1.0 \mathrm{M} \mathrm{NaOH}$ and subsequently placed on a shaker at 100 r.p.m. at room temperature for $18 \mathrm{~h}$. The solution was centrifuged at $10,000 \times g$ for $20 \mathrm{~min}$. Five hundred milliliters of the supernatant of was transferred to a new tube that contained $0.1 \mathrm{ml}$ of concentrated $\mathrm{HCl}$. The tubes were incubated at $4{ }^{\circ} \mathrm{C}$ for $4 \mathrm{~h}$ to precipitate the lignin thioglycolic acid, followed by centrifugation at $13,000 \times g$ for $20 \mathrm{~min}$ at $4{ }^{\circ} \mathrm{C}$ (5404EP320017, Eppendorf, Germany). The precipitate was then dissolved in a $1: 100 \mathrm{ml}$ volume of $1.0 \mathrm{M} \mathrm{NaOH}$. Absorbance was then measured at $280 \mathrm{~nm}$ using ultraviolet spectrophotometry (UV-1800, Japan), with $1.0 \mathrm{M} \mathrm{NaOH}$ used as a blank. The data were expressed on a fresh weight basis and three biological replicates were used for each sample.

\section{RNA isolation, library construction, and sequencing}

The total RNA from the juice sacs was isolated according to the methods of Liu et al. ${ }^{40}$, while RNA from calli was isolated with TRIzol reagent (Aidlab Biotechnologies Co., Ltd, Beijing, China). The RNA from the HR, HB, KP, and FH fruit juice sacs at 55, 85, 115, 145, and 205 DPA was extracted, with three biological replicates included per sample. The following procedures were performed by Novogene Bioinformatics Technology, China. RNA integrity was assessed using a RNA Nano 6000 Assay Kit of a Bioanalyzer 2100 system (Agilent Technologies, CA, USA). A total amount of $3 \mu \mathrm{g}$ of RNA per sample was used as input material for the RNA sample preparations. Sequencing libraries were generated using a NEBNext Ultra RNA Library Prep Kit for Illumina (NEB, USA) following the manufacturer's recommendations and index codes were added to attribute sequences to each sample. The constructed RNA libraries were sequenced on an Illumina HiSeq 2500 platform in pairedend mode, with a read length of $150 \mathrm{bp}$.

\section{RNA-seq data analysis}

Quality control of the raw sequencing data was performed with FastQC. Adapters were removed from reads and the data were cleaned based on phred scores using fastp. The clean data were mapped to the pummelo genome $^{31}$. Read counts for each gene in each sample were extracted with the edgeR program. The FPKM of each gene was calculated based on its definition ${ }^{41}$. Differential expression analysis was performed using DESeq v1.18.0 in $\mathrm{R}$ v3.5.1 $1^{42}$. We then used KOBAS software to test the statistical enrichment of DEGs in KEGG pathways. Gene Ontology (GO) enrichment analysis of DEGs was implemented by GOseq v1.38.0, in which gene length bias was corrected. GO terms with corrected $P$-values $(\alpha=0.05)$ were considered significantly enriched for those DEGs ${ }^{43}$.
For correlation analyses, Spearman's rank correlations of the lignin content and FPKM of all the genes expressed in the $\mathrm{KP}, \mathrm{HB}$ and $\mathrm{HR}$ pummelo juice sacs at the five developmental stages was calculated using the pspearman package. Genes with a correlation coefficient to lignin content ratio higher than 0.75 and a significant $P$-value $(\alpha=0.05)$ were selected.

\section{CDNA synthesis and quantitative real-time PCR}

A total of $0.5 \mu \mathrm{g}$ of RNA was used for CDNA synthesis with a RevertAid First Strand cDNA Synthesis Kit (Thermo Fisher Scientific, USA). qRT-PCR was performed with a Roche LightCycler 480 system in conjunction with 23 LightCycler 480 SYBR Green Master Mix (Roche, USA), as described by Lu et al. ${ }^{44}$. The primers designed for qRT-PCR are listed in Supplementary Table S8 and are based on pummelo database gene sequences ${ }^{31}$. The actin gene was used as previously reported ${ }^{26}$. The qRT-PCR data were analyzed using the $2^{-\Delta C t}$ analysis method.

\section{Gene cloning}

The full-length coding DNA sequence (CDS) and $2.1 \mathrm{~kb}$ promoter region of $C g M Y B 58$ and the promoter of lignin biosynthetic genes were amplified from the cDNA and DNA of various citrus cultivars. The sequences of the primers used are listed in Supplementary Table S8. Sequence results were obtained from $\sim 20$ and 10 clones amplified from cDNA and DNA templates, respectively.

\section{Phylogenetic analysis}

The online version of ClustalW (https://www.genome. jp/tools-bin/clustalw) was utilized for multiple alignment of the amino acid sequences of CgMYB58 and the corresponding sequences of other species. Conserved domains were extracted using gblocks (http://www. phylogeny.fr/one_task.cgi?task_type=gblocks). MEGA 7.0 was subsequently used to construct a phylogenetic tree according to the maximum likelihood method. There were 1000 bootstrap replications and values higher than 50 for each node are presented in the tree.

\section{Subcellular localization analysis}

The $C g M Y B 58$ CDS without a stop codon was fused to green fluorescent protein (GFP) within a pM999 vector. Citrus (C. limon Burm "Eureka lemon") mesophyll protoplasts were extracted carefully for transient transformation. Plasmids of 35S:CgMYB58-GFP and 35S:OsGhd7RFP (nuclear marker) and pM999 empty vector plasmids were mixed together equally. These plasmid mixtures were then transferred into separate protoplasts. After $24 \mathrm{~h}$, the florescence images were scanned via a confocal laser-scanning microscope (TCS SP2, Leica, Wetzlar, Germany). 


\section{Transient transformation of CgMYB58}

The CgMYB58 overexpression construct (vector: pH7WG2D) and a pH7WG2D empty vector used as a control were introduced into Agrobacterium tumefaciens strain GV3101 separately. The GV3101 cells containing the constructs were suspended in infiltration buffer (50 mg of glucose, $1 \mathrm{ml}$ of $50 \mathrm{mM} \mathrm{MES,} 1 \mathrm{ml}$ of $\mathrm{Na}_{3} \mathrm{PO}_{4}$, and $1 \mu \mathrm{l}$ of $1 \mathrm{M}$ acetosyringone per $10 \mathrm{ml}$ of buffer) at a concentration of $\mathrm{OD}_{600}=0.8$, and then injected into the mesocarp of KP and HR pummelo fruits. GFP signals were captured by an inversion fluorescence microscope (Olympus SZX7, Japan) equipped with a light source (H150). Twelve fruits were transformed per treatment.

\section{Stable transformation of CgMYB58 in citrus calli}

The CDS of $C g M Y B 58$ was isolated from HR and cloned into a pH7WG2D overexpression vector. The vector was then introduced into the A. tumefaciens strain EHA105 before it was transformed into calli of RM grapefruit $(C$. paradisi). The citrus callus transformation and growth conditions were performed according to the methods of $\mathrm{Li}$ et al. ${ }^{45}$, with minor modifications. Each transgenic callus was cultured in $500 \mathrm{ml}$ of MT (Murashige and Tucker) media with vitamins (Coolaber Science \& Technology, Beijing, China), supplemented with $80 \mu \mathrm{l}$ of $50 \mathrm{mg}$ $\mathrm{ml}^{-1}$ hygromycin B, whereas without hygromycin B was used for RM (the WT control). Each CgMYB58 overexpression line and RM callus were subcultured six to seven times before analysis.

\section{Dual luciferase transcriptional activity assay}

The dual LUC transcriptional activity assay procedure was modified from that of a previous study ${ }^{44}$. DNA sequences upstream of the translational start codon were amplified by PCR from genomic DNA of HR to generate 1062, 1101, 1298, and $939 \mathrm{bp}$ promoter fragments for CgPAL1, CgPAL2, Cg4CL1, and CgC3H, respectively. The amplified fragments were subsequently inserted into a pGreenII 0800-LUC reporter vector, which was then introduced into A. tumefaciens GV3101 (pSoup-p19) competent cells. The effector vector was a CgMYB58 overexpression (vector: pH7WG2D) construct, and a pH7WG2D empty vector was used as a control. The constructs were also introduced into GV3101 (pSoupp19). The GV3101 cells containing effectors and reporters were mixed to a proportion of 4:1 and then injected into leaves of Nicotiana benthamiana. LUC activities were analyzed according to the methods of $\mathrm{Lu}$ et al. ${ }^{44}$ at 2.5 days after injection.

\section{Yeast one-hybrid assay analysis}

Y1H assays were carried out using a Matchmaker Gold Yeast One-Hybrid system kit (Clontech, USA) and modified according to the methods of $\mathrm{Lu}$ et $\mathrm{al}^{44}$. The promoter fragments of $C g P A L 2$ and $C g C 3 H$ were cloned into a pAbAi vector to produce pAbAi-CgPAL2P and pAbAi-CgC3HP bait constructs, respectively. The bait plasmids were then linearized and integrated into $\mathrm{Y} 1 \mathrm{H}$ Gold yeast, after which they were selected with synthetic dextrose media lacking uracil. The CgMYB58 coding sequences were ligated into pGADT7 to generate an ADCgMYB58 construct. pGADT7 empty vectors (ADpGADT7), serving as negative controls, were then transferred separately into yeast cells containing bait constructs. The transformed yeast cells were diluted with 0 , $400 \times$, and $500 \times$ dilution series of aureobasidin A (AbA) and dotted on SD plates lacking leucine. The cells grew on both types of media containing prey proteins to allow the interaction of bait sequences.

\section{Electrophoretic mobility shift assays}

EMSA assays were performed as described previously $^{26,46}$. pMAL-c5x-CgMYB58 (with maltose binding protein tag) without a stop codon was expressed and purified as described by Lu et al. ${ }^{44}$. The $25-27$ bp probes containing the $\mathrm{AC}$ elements in the promoters of CgPAL1, $C g P A L 2, C g 4 C L 1$, and $C g C 3 H$ were extracted and used as reference sequences to synthesize probes. 5'-FAM-labeled oligonucleotide probes were synthesized and labeled by Shanghai Sangon Company (Shanghai, China). The same oligonucleotides without labels were used as cold competitors. To perform binding reactions, the binding solution $(0.1 \% \mathrm{NP}-40,1 \mathrm{mM}$ benzamidine, $0.5 \mathrm{mM}$ phenylmethylsulfonyl fluoride, $0.5 \mathrm{mM}$ dithiothreitol, $50 \mu \mathrm{g} \mathrm{ml}^{-1}$ bovine serum albumin, $100 \mathrm{ng} \mu^{-1}$ poly(dI$\mathrm{dC})), 2 \mu \mathrm{l}\left(0.5 \mathrm{mg} \mathrm{ml}^{-1}\right)$ of purified maltose binding protein tag fused CgMYB58 and $1 \mu \mathrm{l}$ of the 5'-FAM-labeled probe $\left(10 \mu \mathrm{mol}^{-1}\right)$ were mixed together and incubated at $4{ }^{\circ} \mathrm{C}$ for $45 \mathrm{~min}$. For competition assays, the unlabeled oligonucleotides were incubated with protein and binding buffer at $4{ }^{\circ} \mathrm{C}$ for $45 \mathrm{~min}$. Afterward, $1 \mu \mathrm{l}$ of the $5^{\prime}$-FAMlabeled probe $\left(10 \mu \mathrm{mol}^{-1}\right)$ was added and incubated at $4{ }^{\circ} \mathrm{C}$ for $45 \mathrm{~min}$. The samples were then loaded onto a prerun $6 \%$ polyacrylamide gel. Electrophoresis was performed at $4{ }^{\circ} \mathrm{C}$ using $0.5 \times$ Tris-borate-EDTA as an electrophoresis buffer in the dark for $1 \mathrm{~h}$. Gel images were acquired using an Amersham Imager 600 (GE Healthcare, Tokyo, Japan).

\section{IAA and $A B A$ treatments of pummelo juice sacs}

The ABA- and auxin-responsive elements of gene promoters were predicted by the PlantCARE online database (http://bioinformatics.psb.ugent.be/webtools/plantcare/ html/). The juice sacs of the KP, HB, and HR pummelo cultivars were collected at 145 DPA in 2019 and cultured in murashige and skoog media (4.43 g of murashige and skoog powder, $100 \mathrm{~g}$ of sucrose, and $7 \mathrm{~g}$ of agar per liter of medium), with IAA and ABA at a final concentration of 
$0.5 \mathrm{mM}$. After being cultured for 50 days on the media, the samples were collected for the evaluation of gene expression and lignin content. High-performance liquid chromatography-grade IAA and ABA standards were purchased from Coolaber Science \& Technology (Beijing, China).

\section{Data analysis and software used}

Metabolite and gene expression profiles were processed with Excel and GraphPad Prism 7. Heatmaps were processed by $\mathrm{R}$ with the gplot and pheatmap packages. Duncan's multiple comparison test and Student's $t$-test were conducted in conjunction with analysis of variance in SAS (SAS Institute, Inc., USA).

\section{Acknowledgements}

This study was supported by the National Key Research and Development Program of China (2018YFD1000204) and the National Natural Science Foundation of China (31672102).

\section{Author contributions}

M.Y.S. performed the experiments and wrote the manuscript. X.L. performed the experiments. H.P.Z. and H.B.Y. analyzed the RNA-seq data. Z.Y.H., J.J.C., J.F., W.H.Y., Y.W.J., and J.L.Y. provided technical assistance and research guidance. C. H.D. and J.X. designed the research. C.H.D., J.X., and J.L.Y. revised the manuscript.

\section{Conflict of interest}

The authors declare that they have no conflict of interest.

Supplementary Information accompanies this paper at (https://doi.org/ 10.1038/s41438-020-00360-7).

Received: 11 April 2020 Revised: 29 May 2020 Accepted: 3 June 2020 Published online: 01 September 2020

\section{References}

1. Boudet, A. M., Kajita, S., Grima-pettenati, J. \& Goffner, D. Lignins and lignocellulosics: a better control of synthesis for new and improved uses. Trends Plant Sci. 8, 576-581 (2003).

2. Raes, J. Genome-wide characterization of the lignification toolbox in Arabidopsis. Plant Physiol. 133, 1051-1071 (2003).

3. Zhao, Q. \& Dixon, R. A. Transcriptional networks for lignin biosynthesis: more complex than we thought? Trends Plant Sci. 16, 227-233 (2011).

4. Vanholme, R., Demedts, B., Morreel, K., Ralph, J. \& Boerjan, W. Lignin biosynthesis and structure. Plant Physiol. 153, 895-905 (2010).

5. $\mathrm{Xu}, \mathrm{Q}$. et al. Activator- and repressor-type MYB transcription factors are involved in chilling injury induced flesh lignification in loquat via their interactions with the phenylpropanoid pathway. J. Exp. Bot. 65, 4349-4359 (2014).

6. Zeng, J. et al. EjAP2-1, an AP2/ERF gene, is a novel regulator of fruit lignification induced by chilling injury, via interaction with EjMYB transcription factors. Plant Biotechnol. J. 13, 1325-1334 (2015).

7. Xue, C. et al. PbrMYB169 positively regulates lignification of stone cells in pear fruit. J. Exp. Bot. 70, 1801-1814 (2019).

8. Xue, C. et al. PbrmiR397a regulates lignification during stone cell development in pear fruit. Plant Biotechnol. J. 17, 103-117 (2019).

9. Wu, J., Pan, T., Guo, Z. \& Pan, D. Specific lignin accumulation in granulated juice sacs of Citrus maxima. Agr. Food Chem. 62, 12082-12089 (2014).

10. Jia, N. et al. Citrus sinensis MYB transcription factors CsMYB330 and CsMYB308 regulate fruit juice sac lignification through fine-tuning expression of the Cs4CL1 gene. Plant Sci. 277, 334-343 (2018).

11. Zhao, Q. Lignification: flexibility, biosynthesis and regulation. Trends Plant Sci. 21, 713-721 (2016).
12. Taylor-Teeples, M. et al. An Arabidopsis gene regulatory network for secondary cell wall synthesis. Nature $\mathbf{5 1 7}$, 571-575 (2015).

13. Chen, H. et al. Hierarchical transcription factor and chromatin binding network for wood formation in Populus trichocarpa. Plant Cell 31, 602-626 (2019).

14. Rao, X. et al. Gene regulatory networks for lignin biosynthesis in switchgrass (Panicum virgatum). Plant Biotechnol. J. 17, 580-593 (2018).

15. Zhong, R., Lee, C., Zhou, J., Mccarthy, R. L. \& Ye, Z. A battery of transcription factors involved in the regulation of secondary cell wall biosynthesis in Arabidopsis. Plant cell 20, 2763-2782 (2008)

16. Zhou, J., Lee, C., Zhong, R. \& Ye, Z. H. MYB58 and MYB63 are transcriptional activators of the lignin biosynthetic pathway during secondary cell wall formation in Arabidopsis. Plant Cell 21, 248-266 (2009).

17. Wang, P. et al. Ethylene signal transduction elements involved in chilling injury in non-climacteric loquat fruit. J. Exp. Bot. 61, 179-190 (2010).

18. $\mathrm{Xu}$, Q. et al. A NAC transcription factor, EjNAC1, affects lignification of loquat fruit by regulating lignin. Postharvest Biol. Tec. 102, 25-31 (2015).

19. Wang, S. et al. Characterization and metabolic diversity of flavonoids in citrus species. Sci. Rep. 7, 1-10 (2017).

20. Chen, J. et al. Cit 1,2RhaT and two novel CitdGlcTs participate in flavor-related flavonoid metabolism during citrus fruit development. J. Exp. Bot. 70, 2759-2771 (2019).

21. Zhang, H. et al. Comprehensive comparative analysis of volatile compounds in citrus fruits of different species. Food Chem. 230, 316-326 (2017).

22. He, D. et al. Simultaneous determination of flavanones, hydroxycinnamic acids and alkaloids in citrus fruits by HPLC-DAD-ESI/MS. Food Chem. 127, 880-885 (2011).

23. Xu, C. J., Fraser, P. D., Wang, W. J. \& Bramley, P. M. Differences in the carotenoid content of ordinary citrus and lycopene-accumulating mutants. J. Agric. Food Chem. 54, 5474-5481 (2006).

24. Luo, T. et al. Distinct carotenoid and flavonoid accumulation in a spontaneous mutant of Ponkan (Citrus reticulata Blanco) results in yellowish fruit and enhanced postharvest resistance. J. Agr. Food Chem. 63, 8601-8614 (2015).

25. Yan, F. et al. Largely different carotenogenesis in two pummelo fruits with different flesh colors. PLoS ONE 13, No. e0200320 (2018).

26. Zhu, F. et al. An R2R3-MYB transcription factor represses the transformation of a- and $\beta$-branch carotenoids by negatively regulating expression of $\mathrm{CrBCH} 2$ and CrNCED5 in flavedo of Citrus reticulate. New Phytol. 216, 178-192 (2017).

27. Zhang, J. et al. Identification of microRNAs correlated with citrus granulation based on bioinformatics and molecular biology analysis. Postharvest Biol. Tec. 118, 59-67 (2016).

28. Yao, S., Cao, Q., Xie, J., Deng, L. \& Zeng, K. Alteration of sugar and organic acid metabolism in postharvest granulation of Ponkan fruit revealed by transcriptome profiling. Postharvest Biol. Tec. 139, 2-11 (2018).

29. Wang, X. et al. Effects of granulation on organic acid metabolism and its relation to mineral elements in Citrus grandis juice sacs. Food Chem. 145, 984-990 (2014).

30. Jia, N. et al. Citrus sinensis MYB transcription factor CsMYB85 induce fruit juice sac lignification through interaction with other CsMYB transcription factors. Front. Plant Sci. 10, 1-12 (2019).

31. Wang, X. et al. Genomic analyses of primitive, wild and cultivated citrus provide insights into asexual reproduction. Nat. Genet. 49, 765-772 (2017).

32. Zhong, R., Richardson, E. A. \& Ye, Z. H. The MYB46 transcription factor is a direct target of SND1 and regulates secondary wall biosynthesis in Arabidopsis. Plant Cell 19, 2776-2792 (2007).

33. Steenackers, W. et al. The allelochemical MDCA inhibits lignification and affects auxin homeostasis. Plant Physiol. 172, 874-888 (2016).

34. Khadr, A. et al. Exogenous abscisic acid suppresses the lignification and changes the growth, root anatomical structure and related gene profiles of carrot. Acta Biochim. Biophys. Sin. 52, 97-100 (2020).

35. Gui, J. et al. Phosphorylation of LTF1, an MYB transcription factor in Populus, acts as a sensory switch regulating lignin biosynthesis in wood cells. Mol. Plant 8, 1-13 (2019).

36. $\mathrm{Xu}$, L. et al. Lignin metabolism has a central role in the resistance of cotton to the wilt fungus Verticillium dahliae as revealed by RNA-Seqdependent transcriptional analysis and histochemistry. J. Exp. Bot. 62, 5607-5621 (2011).

37. $\mathrm{Fu}, \mathrm{X}$. et al. Plastid structure and carotenogenic gene expression in red- and white-fleshed loquat (Eriobotrya japonica) fruits. J. Exp. Bot. 63, 341-354 (2012).

38. Wang, Y. et al. Auxin regulates anthocyanin biosynthesis through the Aux IAA-ARF signaling pathway in apple. Hortic. Res. 5, 59 (2018). 
39. $L i, X$. et al. The use of toluidine blue staining combined with paraffin sectioning and the optimization of freeze-thaw counting methods for analysing root-knot nematodes in tomato. Hort. Env. Biotechnol. 58, 620-626 (2017).

40. Liu, Y. Efficient isolation of RNA from fruit peel and pulp of ripening nave orange (Citrus sinensis Osbeck). J. Huazhong Agric. Uni. 25, 300-304 (2006).

41. Trapnell, C. et al. Transcript assembly and quantification by RNA-Seq reveals unannotated transcripts and isoform switching during cell differentiation. Nat. Biotechnol. 28, 516-520 (2010).

42. Wang, L., Feng, Z., Wang, X., Wang, X. \& Zhang, X. DEGseq: an R package for identifying differentially expressed genes from RNA-seq data. Bioinformatics 26, 136-138 (2010).
43. Young, M. D., Wakefield, M. J., Smyth, G. K. \& Oshlack, A. Gene ontology analysis for RNA-seq: accounting for selection bias. Genome Biol. 11, R14 (2010).

44. Lu, S. et al. The citrus transcription factor CsMADS6 modulates carotenoid metabolism by directly regulating carotenogenic genes. Plant Physiol. 176, 2657-2676 (2018).

45. Li, D. D., Shi, W. \& Deng, X. X. Agrobacterium-mediated transformation of embryogenic calluses of Ponkan mandarin and the regeneration of plants containing the chimeric ribonuclease gene. Plant Cell Rep. 21, 153-156 (2002).

46. Huang, D. et al. Subfunctionalization of the Ruby2-Ruby1 gene cluster during the domestication of citrus. Nat. Plants 4, 930-941 (2018). 